\title{
Search for a Hypothetical 16.7 MeV Gauge Boson and Dark Photons in the NA64 Experiment at CERN
}

D. Banerjee, ${ }^{4}$ V. E. Burtsev, ${ }^{11}$ A. G. Chumakov, ${ }^{11}$ D. Cooke, ${ }^{13}$ P. Crivelli, ${ }^{13}$ E. Depero, ${ }^{13}$ A. V. Dermenev, ${ }^{5}$ S. V. Donskov, ${ }^{10}$ R. R. Dusaev, ${ }^{11}$ T. Enik, ${ }^{2}$ N. Charitonidis, ${ }^{3}$ A. Feshchenko, ${ }^{2}$ V. N. Frolov, ${ }^{2}$ A. Gardikiotis, ${ }^{9}$ S. G. Gerassimov, ${ }^{6,8}$ S. N. Gninenko, ${ }^{5,}{ }^{*}$ M. Hösgen, ${ }^{1}$ M. Jeckel, ${ }^{3}$ A. E. Karneyeu, ${ }^{5}$ G. Kekelidze, ${ }^{2}$ B. Ketzer, ${ }^{1}$ D. V. Kirpichnikov, ${ }^{5}$ M. M. Kirsanov, ${ }^{5}$ I. V. Konorov, ${ }^{6,8}$ S. G. Kovalenko, ${ }^{12}$ V. A. Kramarenko, ${ }^{2,7}$ L. V. Kravchuk, ${ }^{5}$ N. V. Krasnikov, ${ }^{5}$ S. V. Kuleshov, ${ }^{12}$ V. E. Lyubovitskij, ${ }^{11,12}$ V. Lysan, ${ }^{2}$ V. A. Matveev, ${ }^{2}$ Yu. V. Mikhailov, ${ }^{10}$ D. V. Peshekhonov, ${ }^{2}$ V. A. Polyakov, ${ }^{10}$ B. Radics, ${ }^{13}$ R. Rojas, ${ }^{12}$ A. Rubbia, ${ }^{13}$ V. D. Samoylenko, ${ }^{10}$ V. O. Tikhomirov, ${ }^{6}$ D. A. Tlisov, ${ }^{5}$ A. N. Toropin, ${ }^{5}$ A. Yu. Trifonov, ${ }^{11}$ B. I. Vasilishin, ${ }^{11}$ G. Vasquez Arenas, ${ }^{12}$ P. V. Volkov, ${ }^{2,7}$ V. Volkov, ${ }^{7}$ and P. Ulloa ${ }^{12}$

(NA64 Collaboration)

\author{
${ }^{1}$ Universität Bonn, Helmholtz-Institut für Strahlen-und Kernphysik, 53115 Bonn, Germany \\ ${ }^{2} J o i n t$ Institute for Nuclear Research, 141980 Dubna, Russia \\ ${ }^{3}$ CERN, European Organization for Nuclear Research, CH-1211 Geneva, Switzerland \\ ${ }^{4}$ University of Illinois at Urbana Champaign, Urbana, 61801-3080 Illinois, USA \\ ${ }^{5}$ Institute for Nuclear Research, 117312 Moscow, Russia \\ ${ }^{6}$ P.N. Lebedev Physics Institute, 119991 Moscow, Russia \\ ${ }^{7}$ Skobeltsyn Institute of Nuclear Physics, Lomonosov Moscow State University, 119991 Moscow, Russia \\ ${ }^{8}$ Technische Universität München, Physik Department, 85748 Garching, Germany \\ ${ }^{9}$ Physics Department, University of Patras, 26504 Patras, Greece \\ ${ }^{10}$ State Scientific Center of the Russian Federation Institute for High Energy Physics of National Research Center \\ "Kurchatov Institute" (IHEP), 142281 Protvino, Russia \\ ${ }^{11}$ Tomsk State Pedagogical University, 634061 Tomsk, Russia \\ ${ }^{12}$ Universidad Técnica Federico Santa María, 2390123 Valparaíso, Chile \\ ${ }^{13}$ ETH Zürich, Institute for Particle Physics and Astrophysics, CH-8093 Zürich, Switzerland
}

(Received 12 March 2018; published 8 June 2018)

\begin{abstract}
We report the first results on a direct search for a new $16.7 \mathrm{MeV}$ boson $(X)$ which could explain the anomalous excess of $e^{+} e^{-}$pairs observed in the excited ${ }^{8} \mathrm{Be}^{*}$ nucleus decays. Because of its coupling to electrons, the $X$ could be produced in the bremsstrahlung reaction $e^{-} Z \rightarrow e^{-} Z X$ by a $100 \mathrm{GeV} e^{-}$beam incident on an active target in the NA64 experiment at the CERN Super Proton Synchrotron and observed through the subsequent decay into a $e^{+} e^{-}$pair. With $5.4 \times 10^{10}$ electrons on target, no evidence for such decays was found, allowing us to set first limits on the $X-e^{-}$coupling in the range $1.3 \times 10^{-4} \lesssim \epsilon_{e} \lesssim$ $4.2 \times 10^{-4}$ excluding part of the allowed parameter space. We also set new bounds on the mixing strength of photons with dark photons $\left(A^{\prime}\right)$ from nonobservation of the decay $A^{\prime} \rightarrow e^{+} e^{-}$of the bremsstrahlung $A^{\prime}$ with a mass $\lesssim 23 \mathrm{MeV}$.
\end{abstract}

DOI: 10.1103/PhysRevLett.120.231802

The ATOMKI experiment of Krasznahorkay et al. [1] has reported the observation of a $6.8 \sigma$ excess of events in the invariant mass distributions of $e^{+} e^{-}$pairs produced in the nuclear transitions of excited ${ }^{8} \mathrm{Be}^{*}$ to its ground state via internal pair creation. This anomaly can be interpreted as

Published by the American Physical Society under the terms of the Creative Commons Attribution 4.0 International license. Further distribution of this work must maintain attribution to the author(s) and the published article's title, journal citation, and DOI. Funded by SCOAP. the emission of a new protophobic gauge $X$ boson with a mass of $16.7 \mathrm{MeV}$ followed by its $X \rightarrow e^{+} e^{-}$decay assuming that the $X$ has nonuniversal coupling to quarks, coupling to electrons in the range $2 \times 10^{-4} \lesssim \epsilon_{e} \lesssim 1.4 \times$ $10^{-3}$ and the lifetime $10^{-14} \lesssim \tau_{X} \lesssim 10^{-12}$ s [2,3]. It has motivated worldwide theoretical and experimental efforts towards light and weakly coupled vector bosons; see, e.g., Refs. [4-12].

Another strong motivation in the search for a new light boson decaying into an $e^{+} e^{-}$pair is provided by the dark matter puzzle. An intriguing possibility is that in addition to gravity a new effective force between the dark sector and 
visible matter, transmitted by a new vector boson $A^{\prime}$ (dark photon) might exist $[13,14]$. Such $A^{\prime}$ could have a mass $m_{A^{\prime}} \lesssim 1 \mathrm{GeV}$, associated with a spontaneously broken gauged $U(1)_{D}$ symmetry, and would couple to the standard model through kinetic mixing with the ordinary photon, $-\frac{1}{2} \epsilon F_{\mu \nu} A^{\prime \mu \nu}$, parametrized by the mixing strength $\epsilon \ll 1$ [15-17]. For a review see, e.g., Refs. [4,18,19]. A number of previous beam dump [20-34], fixed target [35-37], collider [38-40], and rare particle decay [41-53] experiments have already put stringent constraints on the mass $m_{A^{\prime}}$ and $\epsilon$ of such dark photons excluding, in particular, the parameter space region favored by the $g_{\mu}-2$ anomaly. However, the range of mixing strengths $10^{-4} \lesssim \epsilon \lesssim 10^{-3}$ corresponding to a short-lived $A^{\prime}$ still remains unexplored. In this Letter we report the first results from the NA64 experiment specifically designed for a direct search of the $e^{+} e^{-}$decays of new short-lived particles in the sub-GeV mass range at the CERN Super Proton Synchrotron (SPS) [54-57].

The method of the search for $A^{\prime} \rightarrow e^{+} e^{-}$decays is described in Refs. [54,55]. Its application to the case of the $X \rightarrow e^{+} e^{-}$decay is straightforward. Briefly, a high-energy electron beam is sent into an electromagnetic (e.m.) calorimeter that serves as an active beam dump. Typically the beam electron loses all its shower energy in the dump. If the $A^{\prime}$ exists, due to the $A^{\prime}(X)-e^{-}$coupling it would occasionally be produced by a shower electron (or positron) in its scattering off a nuclei of the dump:

$$
e^{-}+Z \rightarrow e^{-}+Z+A^{\prime}(X) ; \quad A^{\prime}(X) \rightarrow e^{+} e^{-} .
$$

Since the $A^{\prime}$ is penetrating and longer lived, it would escape the beam dump, and subsequently decays into an $e^{+} e^{-}$pair in a downstream set of detectors. The pair energy would be equal to the energy missing from the dump. The apparatus is designed to identify and measure the energy of the $e^{+} e^{-}$ pair in another calorimeter (ECAL). Thus, the signature of the $A^{\prime}(X) \rightarrow e^{+} e^{-}$decay is an event with two e.m.-like showers in the detector: one shower in the dump, and another one in the ECAL with the sum energy equal to the beam energy.

The NA64 setup is schematically shown in Fig. 1. The experiment employs the optimized $100 \mathrm{GeV}$ electron beam from the $\mathrm{H} 4$ beam line in the North Area (NA) of the CERN SPS. Two scintillation counters, $S 1$ and $S 2$, were used for the beam definition, while the other two, $S 3$ and $S 4$, were used to detect the $e^{+} e^{-}$pairs. The detector was equipped with two dipole magnets and a tracker, which was a set of four upstream Micromegas (MM) chambers $(T 1, T 2)$ for the incoming $e^{-}$angle selection and two sets of downstream MM, gas electron multiplier (GEM) stations, and scintillator hodoscopes $(T 3, T 4)$ for measurements of the outgoing tracks $[58,59]$. To enhance the electron identification, the synchrotron radiation (SR) emitted by electrons was used for their tagging allowing us to suppress the initial hadron contamination in the beam $\pi / e^{-} \simeq 10^{-2}$ down to the level $\simeq 10^{-6}[57,60]$. The use of SR detectors (SRD) was a key point for the improvement of the sensitivity compared to the previous electron beam dump searches [24,25]. The dump was a compact e.m. calorimeter WCAL made as short as possible to maximize the sensitivity to short lifetimes while keeping the leakage of particles at a small level. It was followed by the ECAL to measure the energy of the decay $e^{+} e^{-}$pair, which was a matrix of $6 \times 6$ shashlik-type modules [57]. The ECAL has $\simeq 40$ radiation lengths $\left(X_{0}\right)$ and is located at a distance $\simeq 3.5 \mathrm{~m}$ from the WCAL. Downstream of the ECAL the detector was equipped with a high-efficiency veto counter, $V 3$, and a hermetic hadron calorimeter (HCAL) [57] used as a hadron veto and for muon identification with a help of four muon counters, MU1-MU4, located between the HCAL modules. The results reported here were obtained from data samples in which $2.4 \times 10^{10}$ electrons on target $\left(\right.$ EOT) and $3 \times 10^{10}$ EOT were collected with the WCAL of $40 X_{0}$ (with a length of $290 \mathrm{~mm}$ ) and of $30 X_{0}(220 \mathrm{~mm})$, respectively. The events were collected with a hardware trigger requiring in-time energy deposition in the WCAL and $E_{\mathrm{WCAL}} \lesssim 70 \mathrm{GeV}$. Data of these two runs (hereafter called the $40 X_{0}$ and $30 X_{0}$ run) were analyzed with similar

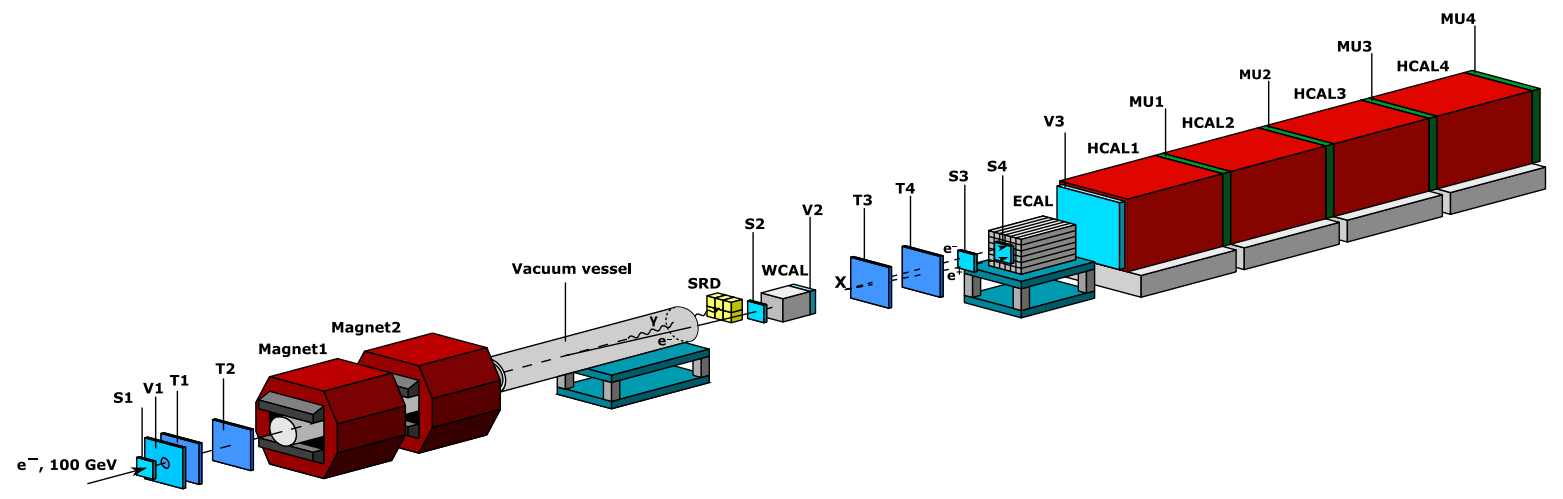

FIG. 1. Schematic illustration of the NA64 setup to search for the $A^{\prime}, X \rightarrow e^{+} e^{-}$decays. 
selection criteria and finally summed up, taking into account the corresponding normalization factors. For the mass range $1 \leq m_{A^{\prime}} \leq 25 \mathrm{MeV}$ and energy $E_{A^{\prime}} \gtrsim 20 \mathrm{GeV}$, the opening angle $\Theta_{e^{+} e^{-}} \simeq 2 m_{A^{\prime}} / E_{A^{\prime}} \lesssim 2 \mathrm{mrad}$ of the decay $e^{+} e^{-}$pair is too small to be resolved in the tracker T3-T4, and the pairs are mostly detected as a single-track e.m. shower in the ECAL.

The candidate events were selected with the following criteria chosen to maximize the signal acceptance and minimize background, using both GEANT4[61,62] based simulations and data. (i) There should be only one track entering the dump. No cuts on reconstructed outgoing tracks were used. (ii) No energy deposition in the $V 2$ counter exceeding about half of the energy deposited by the minimum ionizing particle (MIP). (iii) The signal in the decay counter $S 4$ is consistent with two MIPs. (iv) The sum of energies deposited in the WCAL and ECAL, $E_{\mathrm{tot}}=E_{\mathrm{WCAL}}+E_{\mathrm{ECAL}}$, is equal to the beam energy within the energy resolution of these detectors. According to simulations, at least $30 \%$ of the total energy should be deposited in the ECAL $[63,64]$. (v) The showers in the WCAL and ECAL should start to develop within a few first $X_{0}$. (vi) The lateral and longitudinal shape of the shower in the ECAL are consistent with a single e.m. one. However, for $A^{\prime}$ s with the energy $\lesssim 5 \mathrm{GeV}$, the ECAL shower is poorly described by the single shower shape; hence, the additional cut $E_{\mathrm{ECAL}}>5 \mathrm{GeV}$ was applied. (vii) No significant energy deposited in the $V 3$ and/or HCAL. These cuts were used for rejection of events with hadrons in the final state. As in the previous analyses [56,57], a clean sample of $\simeq 10^{5}$ rare $\mu^{+} \mu^{-}$events produced in the dump was used for the efficiency corrections in the simulations, which do not exceed 20\%. A blind analysis of data was performed, with the signal box defined as $90<E_{\text {tot }}<$ $110 \mathrm{GeV}$ and by using $20 \%$ (100\%) of the data for the selection criteria optimization (background estimate).

There are several processes that can fake the $A^{\prime} \rightarrow e^{+} e^{-}$ signal. Among them, the two most important were expected either from decay chain $K_{S}^{0} \rightarrow \pi^{0} \pi^{0} ; \pi^{0} \rightarrow \gamma e^{+} e^{-}$of $K_{S}^{0}$ produced in the WCAL or from the $\gamma \rightarrow e^{+} e^{-}$conversion of photons from $K_{S}^{0} \rightarrow \pi^{0} \pi^{0} \rightarrow \pi^{0} \rightarrow \gamma \gamma$ decays in the $T 3$ plane or earlier in the beam line. Another background could come from the $K_{S}^{0} \rightarrow \pi^{+} \pi^{-}$hadronic decays that could be misidentified as an e.m. event in the ECAL at the level $\lesssim 2.5 \times 10^{-5}$ evaluated from the measurements with the pion beam. The leading $K^{0}$ can be produced in the dump either by misidentified beam $\pi^{-}, K^{-}$or directly by electrons. The background from the $K_{S}^{0}$ decay chain was estimated by using the direct measurements of the $K_{S}^{0}$ flux from the dump with the following method. It is well known that the $K^{0}$ produced in hadronic reactions is a linear combination of the short- and long-lived components $\left|K^{0}\right\rangle=\left(\left|K_{S}^{0}\right\rangle+\left|K_{L}^{0}\right\rangle\right) / \sqrt{2}$. The flux of $K^{0}$ was evaluated from the measured ECAL + HCAL energy spectrum of long-lived neutral hadrons selected with the requirement of no signal in $V 2$ and $S 4$, taking into account corrections due to the $K_{S}^{0}$ decays in flight. The main fraction of $\simeq 10^{3}$ events observed in the HCAL were neutrons produced in the same processes as $K^{0}$ in the WCAL. According to simulations, $\lesssim 10 \%$ of them were predicted to be other neutral hadrons, i.e., $\Lambda$ and $K^{0}$, that were also included in the data sample. The conservative assumption that $\simeq 100 K^{0}$ were produced allows us to calculate the number of $K_{S}^{0}$ from the dump and simulate the corresponding background from the $K_{S}^{0} \rightarrow$ $\pi^{+} \pi^{-}$and $K_{S}^{0} \rightarrow \pi^{0} \pi^{0} ; \pi^{0} \rightarrow \gamma e^{+} e^{-}$decay chain, which was found to be $\lesssim 0.04$ events per $5.4 \times 10^{10}$ EOT. To cross-check this result, another estimate of this background was used. The true neutral e.m. events, which are presumably photons, were selected with requirements of no charged tracks, i.e., no signals in $V 2$ and $S 4$ counters, plus a single e.m.-like shower in the ECAL defined by cuts (v)-(vii). Three such events were found in the signal box as shown in Fig. 2. Using simulations we calculated that there were $\simeq 150$ leading $K^{0}$ produced in the dump, which is in reasonable agreement with the previous estimate resulting in a conservative $K_{S}^{0}$ background of 0.06 events. The $\mu, \pi$,

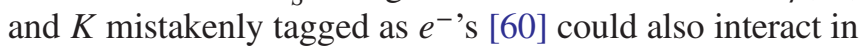
the dump though the $\mu Z \rightarrow \mu Z \gamma$ or $\pi, K$ charge-exchange reactions, accompanied by the poorly detected scattered $\mu$, or secondary hadrons. The misidentified pion could mimic the signal either directly (small fraction of showers that look like an e.m. one) or by emitting a hard bremsstrahlung photon in the last layer of the dump, which then produces an e.m. shower in the ECAL, accompanied by the scattered pion track. Another background can appear from the beam $\pi \rightarrow e \nu$ decays downstream of the WCAL. The latter two backgrounds can pass the selection only due to the $V 2$ inefficiency $\left(\simeq 10^{-4}\right)$, which makes them negligible. The charge-exchange reaction $\pi^{-} p \rightarrow(\geq 1) \pi^{0}+n+\cdots$, which can occur in the last layers of the WCAL with

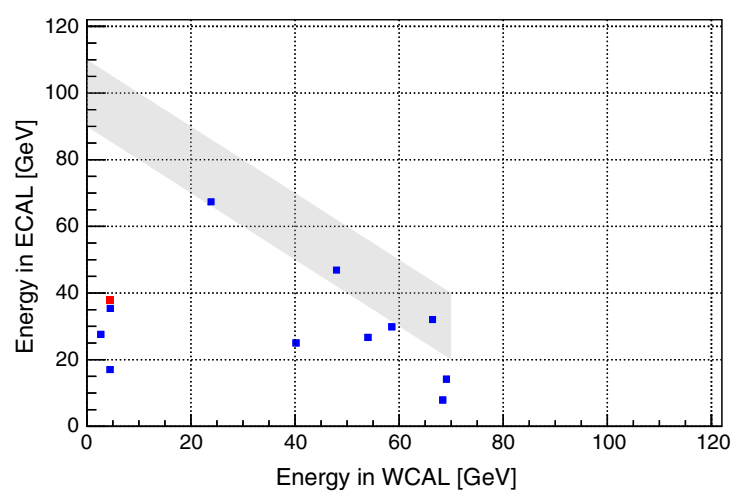

FIG. 2. Distribution of selected e.m. neutral (presumably photon) and signal events in the $\left(E_{\mathrm{WCAL}} ; E_{\mathrm{ECAL}}\right)$ plane from the combined $30 X_{0}$ and $40 X_{0}$ runs. Neutral e.m. events are shown as blue squares. The only signal-like event is shown as a red square. The dashed band represents the signal box. 
TABLE I. Expected numbers of background events in the signal box estimated for $5.4 \times 10^{10}$ EOT.

\begin{tabular}{lr}
\hline \hline Source of background & \multicolumn{1}{c}{ Events } \\
\hline$e^{+} e^{-}$pair production by punchthrough $\gamma$ & $<0.001$ \\
$K_{S}^{0} \rightarrow 2 \pi^{0} ; \pi^{0} \rightarrow \gamma e^{+} e^{-} ; \gamma \rightarrow e^{+} e^{-} ; K_{S}^{0} \rightarrow \pi^{+} \pi^{-}$ & $0.06 \pm 0.034$ \\
$\pi N \rightarrow(\geq 1) \pi^{0}+n+\cdots ; \pi^{0} \rightarrow \gamma e^{+} e^{-} ; \gamma \rightarrow e^{+} e^{-}$ & $0.01 \pm 0.004$ \\
$\pi^{-}$bremsstrahlung in the WCAL, $\gamma \rightarrow e^{+} e^{-}$ & $<0.0001$ \\
$\pi, K \rightarrow e \nu, K_{e 4}$ decays & $<0.001$ \\
$e Z \rightarrow e Z \mu^{+} \mu^{-} ; \mu^{ \pm} \rightarrow e^{ \pm} \nu \nu$ & $<0.001$ \\
Punchthrough $\pi$ & $<0.003$ \\
Total & $0.07 \pm 0.035$ \\
\hline \hline
\end{tabular}

decay photons escaping the dump without interactions and accompanied by poorly detected secondaries, is another source of fake signal. To evaluate this background we used the extrapolation of the charge-exchange cross sections, $\sigma \sim Z^{2 / 3}$, measured on different nuclei [65]. The contribution from the beam kaon decays in flight, $K^{-} \rightarrow e^{-} \nu \pi^{+} \pi^{-}\left(K_{e 4}\right)$, and dimuon production in the dump $e^{-} Z \rightarrow e^{-} Z \mu^{+} \mu^{-}$with either $\pi^{+} \pi^{-}$or $\mu^{+} \mu^{-}$pairs misidentified as e.m. event in the ECAL was found to be negligible.

Table I summarizes the conservatively estimated background inside the signal box, which is expected to be $0.07 \pm 0.034$ events per $5.4 \times 10^{10}$ EOT. The dominant contribution to background is 0.06 events from the $K_{S}^{0}$ decays, with the uncertainty dominated by the statistical error. In Fig. 2, the final distributions of e.m. neutral events, which are presumably photons, and signal candidate events that passed the selection criteria (i)-(iii) and (v)-(vii) are shown in the $\left(E_{\mathrm{ECAL}} ; E_{\mathrm{WCAL}}\right)$ plane. No candidates are found in the signal box. The conclusion that the background is small is confirmed by the data.

The combined $90 \%$ confidence level (C.L.) upper limits for the mixing strength $\epsilon$ were obtained from the corresponding limit for the expected number of signal events, $N_{A^{\prime}}^{90 \%}$, by using the modified frequentist approach, taking the profile likelihood as a test statistic [66-68]. The $N_{A^{\prime}}$ value is given by the sum

$$
N_{A^{\prime}}=\sum_{i=1}^{2} N_{A^{\prime}}^{i}=\sum_{i=1}^{2} n_{\mathrm{EOT}}^{i} \epsilon_{\mathrm{tot}}^{i} n_{A^{\prime}}^{i}\left(\epsilon, m_{A^{\prime}}\right),
$$

where $\epsilon_{\mathrm{tot}}^{i}$ is the signal efficiency in the run $i$ (30 $X_{0}$ or 40 $\left.X_{0}\right)$, and $n_{A^{\prime}}^{i}\left(\epsilon, m_{A^{\prime}}\right)$ is the number of the $A^{\prime} \rightarrow e^{+} e^{-}$decays in the decay volume with energy $E_{A^{\prime}}>30 \mathrm{GeV}$ per EOT, calculated under the assumption that this decay mode is predominant; see, e.g., Eq. (3.7) in Ref. [55]. Each $i$ th entry in this sum was calculated by simulating signal events for the corresponding beam running conditions and processing them through the reconstruction program with the same selection criteria and efficiency corrections as for the data sample from the run $i$. The $A^{\prime}$ efficiency and its systematic error were determined to stem from the overall normalization, $A^{\prime}$ yield, and decay probability, which were the $A^{\prime}$ mass dependent, and also from efficiencies and their uncertainties in the primary $e^{-}(0.85 \pm 0.02)$, $\operatorname{WCAL}(0.93 \pm 0.05), V_{2}(0.96 \pm 0.03), \operatorname{ECAL}(0.93 \pm 0.05)$, $V_{3}(0.95 \pm 0.04)$, and $\operatorname{HCAL}(0.98 \pm 0.02)$ event detection. The latter, shown as example values for the $40 X_{0}$ run, were determined from measurements with the $e^{-}$beam crosschecked with simulations. A detailed simulation of the e.m. shower in the dump [63] with $A^{\prime}$ cross sections was used to calculate the $A^{\prime}$ yield $[64,69,70]$. The $\lesssim 10 \%$ difference between the calculations in Ref. [64] and Refs. $[69,70]$ was accounted for as a systematic uncertainty in $n_{A^{\prime}}\left(\epsilon, m_{A^{\prime}}\right)$. In the overall signal efficiency for each run, the acceptance loss due to pileup ( $\simeq 7 \%$ for $40 X_{0}$ and $\simeq 10 \%$ for $30 X_{0}$ runs) was taken into account and cross-checked using reconstructed dimuon events [57]. The dimuon efficiency corrections $(\lesssim 20 \%)$ were obtained with uncertainty of $10 \%$ and $15 \%$, for the $40 X_{0}$ and $30 X_{0}$ runs, respectively. The total systematic uncertainty on $N_{A^{\prime}}$ calculated by adding all errors in quadrature did not exceed $25 \%$ for both runs. The combined $90 \%$ C.L. exclusion limits on the mixing $\epsilon$ as a function of the $A^{\prime}$ mass is shown in Fig. 3 together with the current constraints from other experiments. Our results exclude the $X$ boson as an explanation for the ${ }^{8} \mathrm{Be}$ anomaly for the $X-e^{-}$coupling $\epsilon_{e} \lesssim 4.2 \times 10^{-4}$ and mass value of

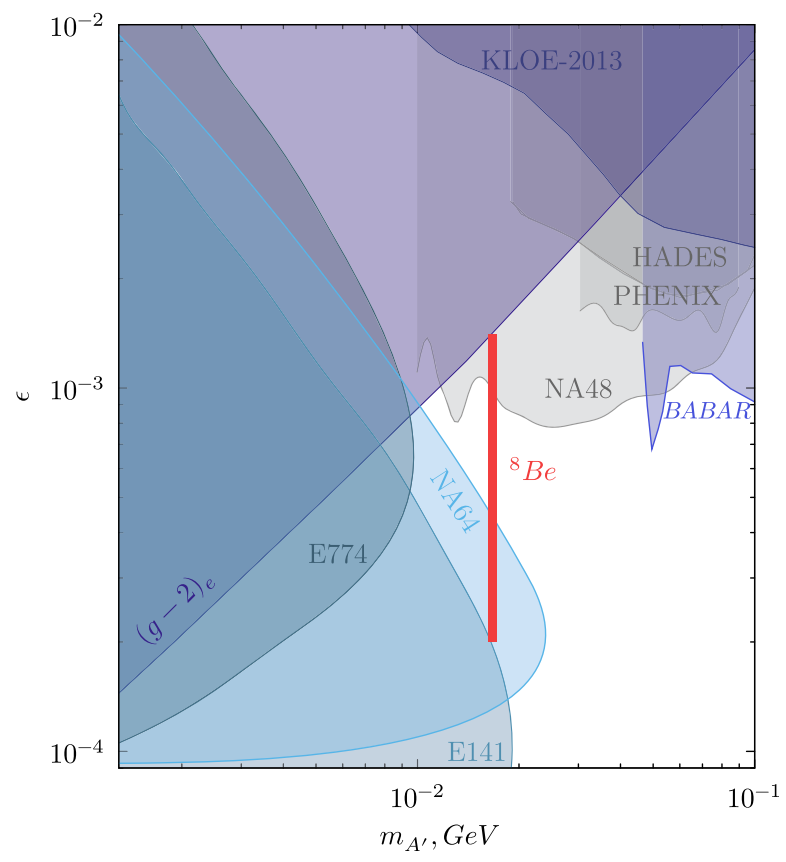

FIG. 3. The $90 \%$ C.L. exclusion areas in the $\left(m_{X} ; \epsilon\right)$ plane from the NA64 experiment (blue area). For the mass of $16.7 \mathrm{MeV}$, the $X-e^{-}$coupling region excluded by NA64 is $1.3 \times 10^{-4}<$ $\epsilon_{e}<4.2 \times 10^{-4}$. The allowed range of $\epsilon_{e}$ explaining the ${ }^{8} \mathrm{Be}$ anomaly (red area) [2,3], constraints on the mixing $\epsilon$ from the experiments E141 [22], E774 [25], BABAR [40], KLOE [45], HADES [47], PHENIX [48], NA48 [50], and bounds from the electron anomalous magnetic moment $(g-2)_{e}$ [71] are also shown. 
$16.7 \mathrm{MeV}$, leaving the still unexplored region $4.2 \times 10^{-4} \lesssim$ $\epsilon_{e} \lesssim 1.4 \times 10^{-3}$ as quite an exciting prospect for further searches.

We gratefully acknowledge the support of the CERN management and staff and the technical staffs of the participating institutions for their vital contributions. We also thank Attila Krasznahorkay for useful discussions about the ATOMKI experiment, and Christophe Menezes Pires and Ondrej Subrt for their help. This work was supported by the HISKP, University of Bonn (Germany), JINR (Dubna), MON and RAS (Russia), SNSF Grant No. 169133 and ETHZ (Switzerland), and Grants FONDECYT No. 1140471 and No. 1150792, Ring ACT1406 and Basal FB0821 CONICYT (Chile). Part of the work on simulations was supported by the RSF Grant No. 14-12-01430.

*Corresponding author Sergei.Gninenko@cern.ch

[1] A. J. Krasznahorkay et al., Phys. Rev. Lett. 116, 042501 (2016).

[2] J. L. Feng, B. Fornal, I. Galon, S. Gardner, J. Smolinsky, T. M. P. Tait, and P. Tanedo, Phys. Rev. Lett. 117, 071803 (2016).

[3] J. L. Feng, B. Fornal, I. Galon, S. Gardner, J. Smolinsky, T. M. P. Tait, and P. Tanedo, Phys. Rev. D 95, 035017 (2017).

[4] M. Battaglieri et al., arXiv:1707.04591.

[5] E. Nardi, C. D. R. Carvajal, A. Ghoshal, D. Meloni, and M. Raggi, Phys. Rev. D 97, 095004 (2018).

[6] J. Kozaczuk, Phys. Rev. D 97, 015014 (2018).

[7] Ch.-W. Chiang and P.-Y.Tseng, Phys. Lett. B 767, 289 (2017).

[8] X. Zhang and G. A. Miller, Phys. Lett. B 773, 159 (2017).

[9] I. Alikhanov and E. A. Paschos, arXiv:1710.10131.

[10] Y. Liang, L.-B. Chen, and C.-F. Qiao, Chin. Phys. C 41, 063105 (2017).

[11] B. Fornal, Int. J. Mod. Phys. A 32, 1730020 (2017).

[12] P. Fayet, Eur. Phys. J. C 77, 53 (2017).

[13] M. Pospelov, A. Ritz, and M. B. Voloshin, Phys. Lett. B 662, 53 (2008).

[14] M. Pospelov, Phys. Rev. D 80, 095002 (2009).

[15] L. B. Okun, Zh. Eksp. Teor. Fiz. 83, 892 (1982) [Sov. Phys. JETP 56, 502 (1982)].

[16] P. Galison and A. Manohar, Phys. Lett. B 136, 279 (1984).

[17] B. Holdom, Phys. Lett. B 166, 196 (1986).

[18] J. Jaeckel and A. Ringwald, Annu. Rev. Nucl. Part. Sci. 60, 405 (2010).

[19] J. Alexander et al., arXiv:1608.08632.

[20] J. D. Bjorken, R. Essig, P. Schuster, and N. Toro, Phys. Rev. D 80, 075018 (2009).

[21] F. Bergsma et al. (CHARM Collaboration), Phys. Lett. 166B, 473 (1986).

[22] E. M. Riordan et al., Phys. Rev. Lett. 59, 755 (1987).

[23] J. D. Bjorken, S. Ecklund, W. R. Nelson, A. Abashian, C. Church, B. Lu, L. W. Mo, T. A. Nunamaker, and P. Rassmann, Phys. Rev. D 38, 3375 (1988).
[24] A. Konaka et al., Phys. Rev. Lett. 57, 659 (1986).

[25] A. Bross, M. Crisler, S. H. Pordes, J. Volk, S. Errede, and J. Wrbanek, Phys. Rev. Lett. 67, 2942 (1991).

[26] M. Davier and H. Nguyen Ngoc, Phys. Lett. B 229, 150 (1989).

[27] C. Athanassopoulos et al. (LSND Collaboration), Phys. Rev. C 58, 2489 (1998).

[28] P. Astier et al. (NOMAD Collaboration), Phys. Lett. B 506, 27 (2001).

[29] S. Adler et al. (E787 Collaboration), Phys. Rev. D 70, 037102 (2004).

[30] R. Essig, R. Harnik, J. Kaplan, and N. Toro, Phys. Rev. D 82, 113008 (2010).

[31] J. Blumlein and J. Brunner, Phys. Lett. B 701, 155 (2011).

[32] S. N. Gninenko, Phys. Lett. B 713, 244 (2012).

[33] J. Blumlein and J. Brunner, Phys. Lett. B 731, 320 (2014).

[34] S. Andreas, C. Niebuhr, and A. Ringwald, Phys. Rev. D 86, 095019 (2012).

[35] S. Abrahamyan et al. (APEX Collaboration), Phys. Rev. Lett. 107, 191804 (2011).

[36] H. Merkel et al., Phys. Rev. Lett. 112, 221802 (2014).

[37] H. Merkel et al. (A1 Collaboration), Phys. Rev. Lett. 106, 251802 (2011).

[38] B. Aubert et al. (BABAR Collaboration), Phys. Rev. Lett. 103, 081803 (2009).

[39] D. Curtin et al., Phys. Rev. D 90, 075004 (2014).

[40] J. P. Lees et al. (BABAR Collaboration), Phys. Rev. Lett. 113, 201801 (2014).

[41] G. Bernardi, G. Carugno, J. Chauveau, F. Dicarlo, M. Dris et al., Phys. Lett. B 166B, 479 (1986).

[42] R. M. Drees et al. (SINDRUM I Collaboration), Phys. Rev. Lett. 68, 3845 (1992).

[43] F. Archilli et al. (KLOE-2 Collaboration), Phys. Lett. B 706, 251 (2012).

[44] S. N. Gninenko, Phys. Rev. D 85, 055027 (2012).

[45] D. Babusci et al. (KLOE-2 Collaboration), Phys. Lett. B 720, 111 (2013).

[46] P. Adlarson et al. (WASA-at-COSY Collaboration), Phys. Lett. B 726, 187 (2013).

[47] G. Agakishiev et al. (HADES Collaboration), Phys. Lett. B 731, 265 (2014).

[48] A. Adare et al. (PHENIX Collaboration), Phys. Rev. C 91, 031901 (2015).

[49] A. V. Artamonov et al. (BNL-E949 Collaboration), Phys. Rev. D 79, 092004 (2009).

[50] J. R. Batley et al. (NA48/2 Collaboration), Phys. Lett. B 746, 178 (2015).

[51] V. V. Dubinina, N. P. Egorenkova, E. A. Pozharova, N. G. Polukhina, V. A. Smirnitsky, and N. I. Starkov, Yad. Fiz. 80, 245 (2017) [Phys. At. Nucl. 80, 461 (2017).

[52] A. Anastasi et al. (KLOE-2 Collaboration), Phys. Lett. B 750, 633 (2015).

[53] A. Anastasi et al. (KLOE-2 Collaboration), Phys. Lett. B 757, 356 (2016).

[54] S. N. Gninenko, Phys. Rev. D 89, 075008 (2014).

[55] S. Andreas et al., arXiv:1312.3309.

[56] D. Banerjee et al. (NA64 Collaboration), Phys. Rev. Lett. 118, 011802 (2017). 
[57] D. Banerjee et al. (NA64 Collaboration), Phys. Rev D 97, 072002 (2018).

[58] D. Banerjee, P. Crivelli, and A. Rubbia, Adv. High Energy Phys. 2015, 105730 (2015).

[59] D. Banerjee et al., Nucl. Instrum. Methods Phys. Res., Sect. A 881, 72 (2018).

[60] E. Depero et al., Nucl. Instrum. Methods Phys. Res., Sect. A 866, 196 (2017).

[61] S. Agostinelli et al. (GEANT4 Collaboration), Nucl. Instrum. Methods Phys. Res., Sect. A 506, 250 (2003).

[62] J. Allison et al., IEEE Trans. Nucl. Sci. 53, 270 (2006).

[63] S. N. Gninenko, N. V. Krasnikov, M. M. Kirsanov, and D. V. Kirpichnikov, Phys. Rev. D 94, 095025 (2016).

[64] S. N. Gninenko, N. V. Krasnikov, M. M. Kirsanov, and D. V. Kirpichnikov, arXiv:1712.05706.
[65] V. N. Bolotov, V. V. Isakov, V. A. Kachanov, D. B. Kakauridze, V. M. Kutyin, Yu. D. Prokoshkin, E. A. Razuvaev, and V. K. Semenov, Nucl. Phys. B85, 158 (1975).

[66] T. Junk, Nucl. Instrum. Methods Phys. Res., Sect. A 434, 435 (1999).

[67] G. Cowan, K. Cranmer, E. Gross, and O. Vitells, Eur. Phys. J. C 71, 1554 (2011).

[68] A. L. Read, J. Phys. G 28, 2693 (2002).

[69] Y. S. Liu, D. McKeen, and G. A. Miller, Phys. Rev. D 95, 036010 (2017).

[70] Y.S. Liu and G. A. Miller, Phys. Rev. D 96, 016004 (2017).

[71] H. Davoudiasl, H. S. Lee, and W. J. Marciano, Phys. Rev. D 89, 095006 (2014). 